\title{
OCORRÊNCIA DE MALÁRIA NO RAMAL DO KM 12, ÁREA RURAL DO MUNICÍPIO DE LÁBREA - AMAZONAS
}

Jhemerson Fernandes Paes ${ }^{(a)}$, Déborah Pereira Linhares da Silva ${ }^{(b)}$, Joiada Moreira da Silva Linhares ${ }^{(\mathrm{c})}$, Tácila Letícia Martins Linhares ${ }^{\text {(d) }}$

(a) Acadêmico de Biomedicina, Centro Universitário do Norte - UNINORTE, jhemersonpaes@ gmail.com

(b) Técnica Administrativa em Educação, Departamento de Ensino, Pesquisa e Extensão do Instituto Federal de Educação, Ciência e Tecnologia do Amazonas - IFAM, campus Lábrea, deborah.silva@ifam.edu.br

(c) Docente EBTT, Instituto Federal de Educação, Ciência e Tecnologia do Amazonas - IFAM, campus Lábrea, e Doutorando do Programa de Pós-Graduação em Desenvolvimento Regional e Meio Ambiente da Universidade Federal de Rondônia - PGDRA / UNIR, joiada.linhares@gmail.com

(d) Dicente do Curso Técnico Integrado ao Ensino Médio em Administração, Instituto Federal de Educação, Ciência e Tecnologia do Amazonas - IFAM, campus Lábrea, tacila.leticia@ gmail.com

\section{EIXO: BACIAS HIDROGRÁFICAS E RECURSOS HÍDRICOS: ANÁLISE, PLANEJAMENTO E GESTÃO}

\section{Resumo}

O objetivo deste estudo foi analisar a ocorrência de malária na comunidade Palheiral (ramal do Km 12), área rural do município de Lábrea - AM, visto que apesar de a malária ser considerada uma doença parasitária de distribuição global, na Amazônia, especialmente no Amazonas, o seu vetor encontra as condições ambientais ideais para sua proliferação. A proposta metodológica se desenvolveu a partir da análise e seleção do material bibliográfico pertinente ao tema e da análise de dados do SIVEP. A ocorrência da malária variou nos anos de 2014 até 2016, ao que se atribui ao as intervenções preventivas da secretaria de saúde no ano 2015. Com este é possível inferir que a abordagem dos problemas ou questões de saúde relacionadas ao ambiente e as características dos locais onde vivem grupos populacionais é fundamental para a compreensão das condições de saúde da população, especialmente da malária na Amazônia.

Palavras chave: malária, habitação, área rural, Lábrea

\section{Introdução}

O Brasil é o país da América do Sul com o maior número de casos registrados de malária, doença parasitária prevalente de distribuição global com um custo "fixo" para o sistema público de saúde. A região norte é considerada área endêmica e o estado do Amazonas se destaca por concentrar o maior número absoluto de casos (BRASIL, 2006).

Estudo realizado por Castro \& Singer (2007) indica que entre os fatores de risco para a transmissão da malária está a implantação de áreas de assentamento, que apresentam maior crescimento no Índice Parasitário Anual (IPA), pois a ocupação do solo associado ao ambiente natural influencia a condição de saúde do ser humano. Neste caso, o parasito da malária encontra as condições ambientais ideais para interação com o vetor e sua proliferação na região amazônica. 
Às condições naturais que subsidiam o desenvolvimento do mosquito transmissor da malária somam-se as particularidades do espaço e tempo, processo que envolve as condições biológicas, ecológicas, socioeconômicas e comportamentais (CASTRO \& SINGER, 2007). Pois as pessoas têm uma relação espacial com o lugar onde vivem e desenvolvem suas atividades (trabalho, lazer e saúde, por exemplo). Essas diferenças, muitas vezes representadas por desigualdades econômicas e sociais, podem indicar quem ali vive e as circunstâncias as quais estão expostas. Por isso Pontes (2003) escreveu que há uma relação entre os lugares e a produção de doenças, que são resultados de um processo histórico, ambiental e social. Desta forma, é possível, através do lugar identificar as condições de habitação e a relação da frequência de algumas doenças, a exemplo da malária. Neste contexto o objetivo deste estudo foi analisar a ocorrência da malária na comunidade Palheiral (ramal do Km 12), área rural do município de Lábrea, Amazonas.

\section{Materiais e Métodos}

O estudo foi desenvolvido no ramal do $\mathrm{Km} \mathrm{12,} \mathrm{na} \mathrm{comunidade} \mathrm{denominada} \mathrm{Palheiral,} \mathrm{assentamento}$ localizado na bacia hidrográfica do rio Paciá, distante 12 quilômetros da área urbana do município de Lábrea, região sul do estado do Amazonas, entre as coordenadas geográficas 6450'33'” de longitude Oeste e 6430'37', de Latitude Sul (Figura 1).

A área de estudo foi escolhida experimentalmente pela proximidade com a área urbana, que poderia facilitar o acesso. Acesso que infelizmente foi dificultado pela condição da estrada até o assentamento, que deixaria a população "isolada" se não fosse o acesso pelo rio Paciá. Nos assentamentos rurais, devido a pouca estrutura e condição socioeconômica dos parceleiros, podem ocorrer mais casos de malária (CASTRO e SINGER, 2007).

Para iniciar a pesquisa foram coletados, analisados e selecionados os materiais bibliográficos (artigos, teses, dissertações, notas e outros) pertinentes ao tema. A pesquisa bibliográfica foi base para aquisição de informações da questão da malária na Amazônia, especificamente no estado do Amazonas.

Foram coletados dados do Sistema de Informação de Vigilância Epidemiológica - SIVEP, referente o período 2005 até 2016, com detalhamento de dados para o ramal do Km 12 do período de 2014 até maio de 2016. Neste sistema, de acesso restrito aos servidores que atuam no serviço de saúde, são registradas as notificações de casos de malária.

Para análise do risco de malária considerou-se o Índice Parasitário Anual para a Região da Amazônia Legal, com graus de risco expressos em valores do IPA: baixo (0,1 a 9,9) médio $(10,0$ a 49,9) e alto (maior ou igual a 50,0 ). 


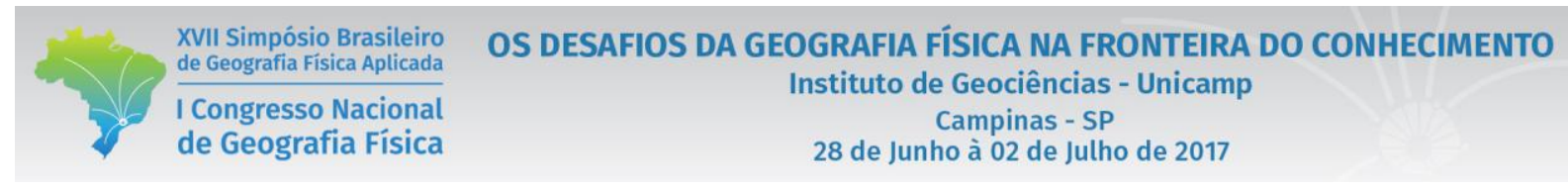

Em campo foram identificadas as propriedades rurais, com registro fotográfico para identificar o tipo de moradia (habitação).

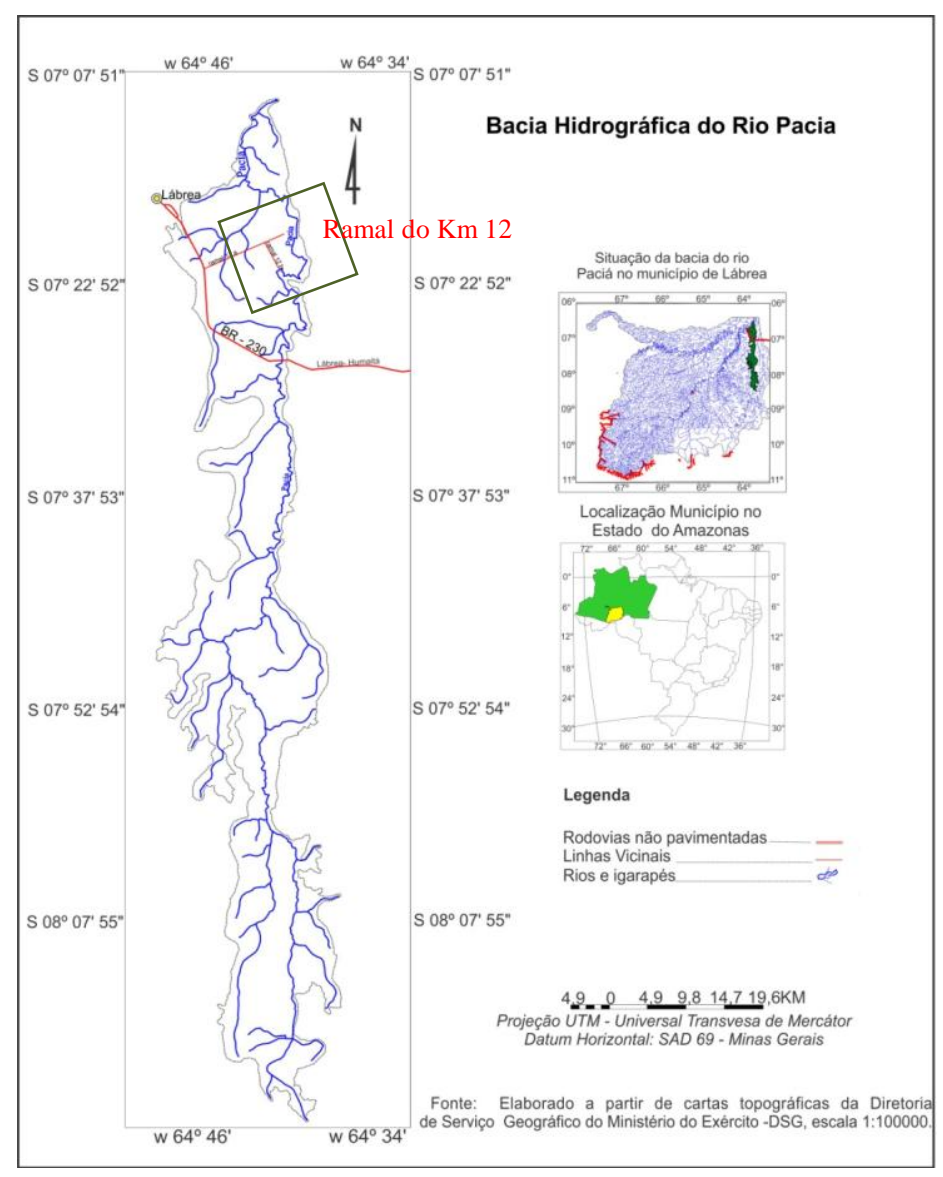

Figura 1 - Bacia Hidrográfica do rio Paciá, onde está localizado o ramal do Km 12.

\section{Resultados e Discussão}

Da análise dos dados constatou-se que nos últimos 10 anos houve 38.627 exames positivos para malária no município de Lábrea. Destes, 36.921 foram classificados como autóctone. Especificamente no ramal do Km 12, em 2014, o Índice Parasitário Anual foi de 1.720, o que representa alto risco de ocorrência de malária (Figura 2).

Da análise dos registros de malária da plataforma SIVEP-Malária, é possível tentar um diagnóstico da situação na comunidade do ramal do Km 12. Através deste foi possível observar que a contagem dos casos não é feita de forma a indicar quantas vezes o um individuo contraiu a patologia, mas sim o total de casos. Com base nas informações desta plataforma, a população da comunidade era de 68 habitantes e os casos 


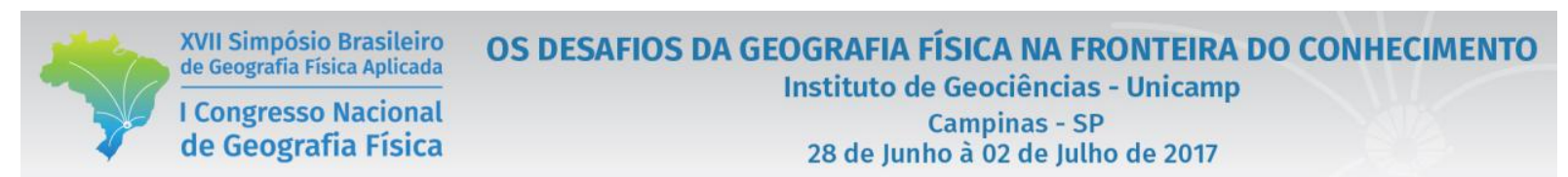

confirmados de malária foram de 117 casos (isto no período de 01/01/2014 até 31/12/2014). O número de casos registrados ultrapassa o quantitativo de habitantes da comunidade do Palheiral.

Outro componente que deve ser destacado é que neste período a plataforma especifica o tipo de protozoário que infectou o individuo, demonstrando assim uma informação acerca da variedade de protozoários causadores da malária na nossa região. Por exemplo, no ano de 2014 foram registrados casos apenas de infecções através dos protozoários Plasmodium vivax e P. falciparum.

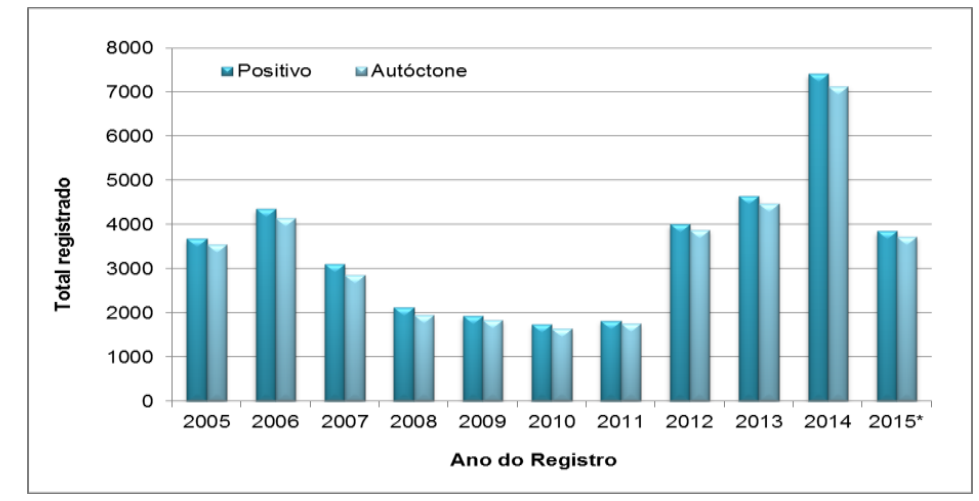

Figura 2. Total de exames positivos de malária de 2005 até 2015 no município de Lábrea - AM.

Fonte: Sistema de Informações de Vigilância Epidemiológica - Notificação de Casos.

Porém, nos últimos dados obtidos do SIVEP-malária, as informações são mais limitadas, o que leva a seguinte proposição, quanto ao quantitativo desta patologia no ramal do Km 12 no período de 2015 e início de 2016, onde a partir do quantitativo de casos e na ausência dos dados referentes à quantidade de habitantes no ano de 2015, pode-se deduzir que os casos de malária diminuíram consideravelmente em relação aos dados de 2014. O que pode ser consequência de campanha de conscientização sobre a doença, que ocorreu de forma assídua no ano 2015 com a distribuição de mosqueteiros, telas protetoras, borrifação de áreas consideradas endêmicas entre outras medidas que podem ter diminuído os casos da doença na comunidade, conforme relatos das ações dos profissionais de saúde.

Nesta análise se considera o método de computar os dados no SIVEP, que é a quantidades de casos totais (e não a quantidade de pessoas infectadas). Para a formulação dos dados do índice parasitário anual (IPA) é feita a contagem de casos totais, ou seja, todas as vezes que o indivíduo contraiu a doença.

Outra questão relevante é a vinculação da malária às características da habitação em área rural, o que se relaciona às condições socioeconômicas e ambientais. A estrutura habitacional das casas localizadas no ramal do Km estão demonstradas na figura 3. Estas reúnem condições que facilitam a proliferação do mosquito, ou seja, com intensa exposição humana. 
O comportamento dos moradores, com exposição mesmo nos horários mais intensos de transmissão, demonstra uma aceitação a condição em que estão inseridos. No ramal não há serviço de saúde, apenas é realizada a coleta de sangue para identificar a doença e tratá-la. Não há serviço preventivo como relatado ter ocorrido no ano 2014.
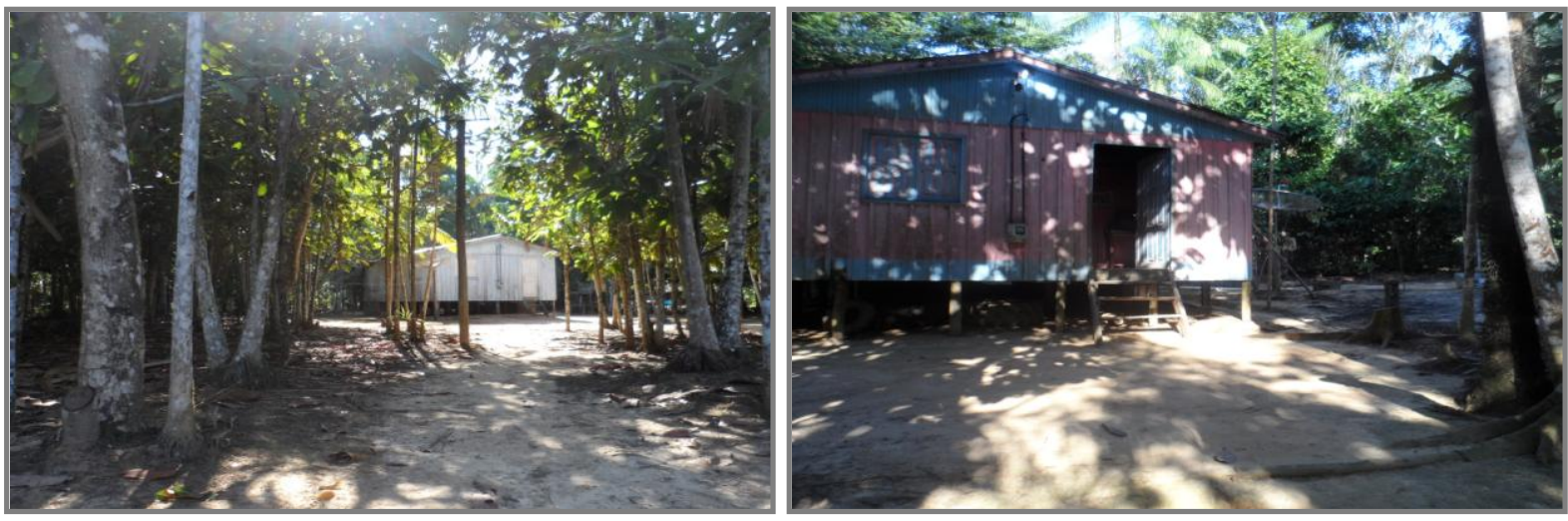

Figura 3 - Unidades habitacionais do ramal do Km 12

\section{Conclusão}

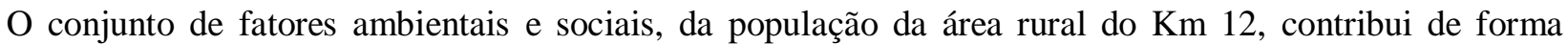
negativa para o IPA, que registra o total de casos, sem considerar o número de casos por indivíduo. O serviço de saúde ofertado à comunidade é deficiente, pois não previne, apenas trata o individuo, o que o deixa debilitado, dada as características da doença. Diante deste contexto é possível inferir que a abordagem dos problemas ou questões de saúde relacionadas ao ambiente e as características dos locais onde vivem grupos populacionais é fundamental para a compreensão das condições de saúde da população.

\section{Referencias}

BRASIL. Ministério da Saúde. Ações de controle da malária: manual para profissionais de saúde na atenção básica. Brasília: Editora do Ministério da Saúde, 2006.

CASTRO, M. C. \& SINGER, B. H. Meio ambiente e saúde: metodologia para análise espacial da ocorrência de malária em projetos de assentamento. R. bras. Est. Pop., São Paulo, v. 24, n. 2, p. 247-262, 2007.

PONTES, J. S.; BORGES, U. N.; PONTES, M. L. F.; LIMA, E. R. V. Espaço, saúde e ambiente: Uma análise espacial da comunidade Santa Clara por meios de técnicas de geoprocessamento. Cadernos do Logepa, V. 2, 116-126, 2003. 